\title{
MYOCARDIAL PERFUSION THROUGH NATURALLY OCCURRING SUBENDOCARDIAL CHANNELS
}

\author{
Daniel Burkhoff, MD, $\mathrm{PhD},{ }^{\mathrm{a}}$ Regan Fulton, MD, $\mathrm{PhD},{ }^{\mathrm{b}}$ Keith Wharton, $\mathrm{MD}, \mathrm{PhD},{ }^{\mathrm{b}}$ Margaret E. Billingham, MD, \\ and Robert Robbins, $\mathrm{MD}{ }^{\mathrm{c}}{ }^{\mathrm{N}}$ New York, N.Y., and Stanford, Calif.
}

Transmyocardial laser revascularization (TMLR) is currently being actively investigated as a therapy for patients with medically refractory angina who cannot be treated by either coronary bypass grafting or percutaneous transluminal coronary angioplasty. ${ }^{1}$. The original idea behind TMLR was to mimic the physiology of myocardial perfusion in reptile hearts by creating channels for blood to flow from the ventricular chamber to directly perfuse the myocardium. However, with elucidation of the anatomic features of alligator hearts, which permit a substantial amount of direct myocardial perfusion to occur, ${ }^{2}$ it has become unclear whether creation of channels that are 1 $\mathrm{mm}$ in diameter and $1 \mathrm{~cm}$ apart can provide perfusion to a substantial amount of myocardium. Results of experiments performed in animals have raised other questions about the physiology of TMLR channels; thus far, no direct evidence has been found to indicate that blood flow through transmyocardial laser channels contributes to myocardial perfusion in mammalian hearts. In this case report we present histologic images that demonstrate naturally occurring subendocardial myocardial channels in a patient with severe coronary artery disease. These unique images reveal a pattern of myocardial viability around the natural channels. The images suggest that direct myocardial perfusion from the chamber is possible but, as for alligator hearts, that a very high channel density would be required to achieve a physiologically meaningful degree of myocardial perfusion. In addition, this patient underwent TMLR with a holmium:yttrium-aluminum-garnet (YAG) laser 2 weeks before his death, and we provide the first reported histologic studies of permanent holmium:YAG channels in human myocardium.

The patient was a 74-year-old man with multiple cardiac risk factors, a remote myocardial infarction, and fourvessel coronary bypass grafting 10 years before his death. Symptoms of heart failure and angina recurred 3 years after the operation. Despite maximal tolerable medical therapy, these symptoms had worsened significantly during the past year. Cardiac catheterization revealed significant left main stenosis and occlusion of the native cir-

From the Department of Medicine, ${ }^{a}$ Columbia University, New York, N.Y., and the Departments of Pathology ${ }^{\mathrm{b}}$ and Cardio-

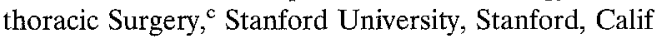

Received for publication Dec. 24, 1996; accepted for publication Jan. 3, 1997.

Address for reprints: Daniel Burkhoff, $\mathrm{MD}, \mathrm{PhD}$, Department of Medicine, Columbia University, 630 W. 168th St., New York, NY 10032.

J Thorac Cardiovasc Surg 1997;114:497-9

Copyright (C) 1997 by Mosby-Year Book, Inc.

$0022-5223 / 97 \$ 5.00+0 \quad \mathbf{1 2 / 5 4 / 8 0 2 3 5}$ cumflex, right coronary, and mid-left anterior descending (LAD) arteries; saphenous vein grafts were occluded, but a left internal thoracic artery graft to the LAD was patent. Echocardiography showed the ejection fraction to be about $40 \%$. He was deemed to be an unsuitable surgical candidate and continued to receive medical therapy. Because of continued worsening of symptoms and the lack of other options, he was referred for treatment with TMLR using a holmium:YAG laser (CardioGenesis Corp., Sunnyvale, Calif.). After consenting to participate in the investigation, he underwent the procedure during which time 24 channels were created with about $1 \mathrm{~cm}$ interchannel spacing in the inferoapical and lateral left ventricular walls. The postoperative course was unremarkable and he was discharged on postoperative day 4 . On postoperative day 13 the patient noted worsening shortness of breath. He died in his sleep the next morning.

Autopsy revealed extensive coronary artery disease with $80 \%$ stenosis of the proximal left main coronary artery, complete occlusion of the LAD distal to the internal thoracic artery anastomosis, and complete occlusion of the proximal circumflex and right coronary arteries. Approximately 24 granulating epicardial scars, each less than $3 \mathrm{~mm}$ in diameter, were identified in the region in which the TMLR was performed. An acute and organizing subendocardial myocardial infarction measuring $8 \times 6 \mathrm{~cm}$ was present in the posterior left ventricular free wall and interventricular septum. The findings of neutrophils, myocardial necrosis, and granulation tissue with early fibrosis were consistent with 3to 14-day-old infarcts in this region. The timing of the LAD occlusion could not be determined from this evaluation.

Fig. 1 shows the typical appearance of TMLR-treated myocardium 2 weeks after surgery. The central core of the channel is filled with organized thrombus surrounded by granulation tissue with associated vascularity, comprising what we have referred to as a channel remnant. The overall appearance of these channel remnants, which we believe are the first published images of subacute holmium:YAG TMLR channels, were similar to what we have seen in a human autopsy specimen from another patient after TMLR treatment with a carbon dioxide laser ${ }^{3}$ and in dog hearts with both holmium:YAG and carbon dioxide lasers.*

Another interesting finding from this autopsy evaluation, shown in Fig. 2, was evidence of naturally occurring subendocardial, endothelium-lined channels or sinusoids. This sample was obtained from the anterior papillary muscle. The extensive fibrosis indicates prior subendocardial myocardial infarction in this region. Most interesting were the circular zones of viable myocytes around the

\footnotetext{
*Fisher PE, Kohmoto T, DeRosa CM, Spotnitz HM, Smith CR, Burkhoff D. Histologic analysis of transmyocardial laser channels: comparison of acute and chronic effects of different lasers. Unpublished data.
} 


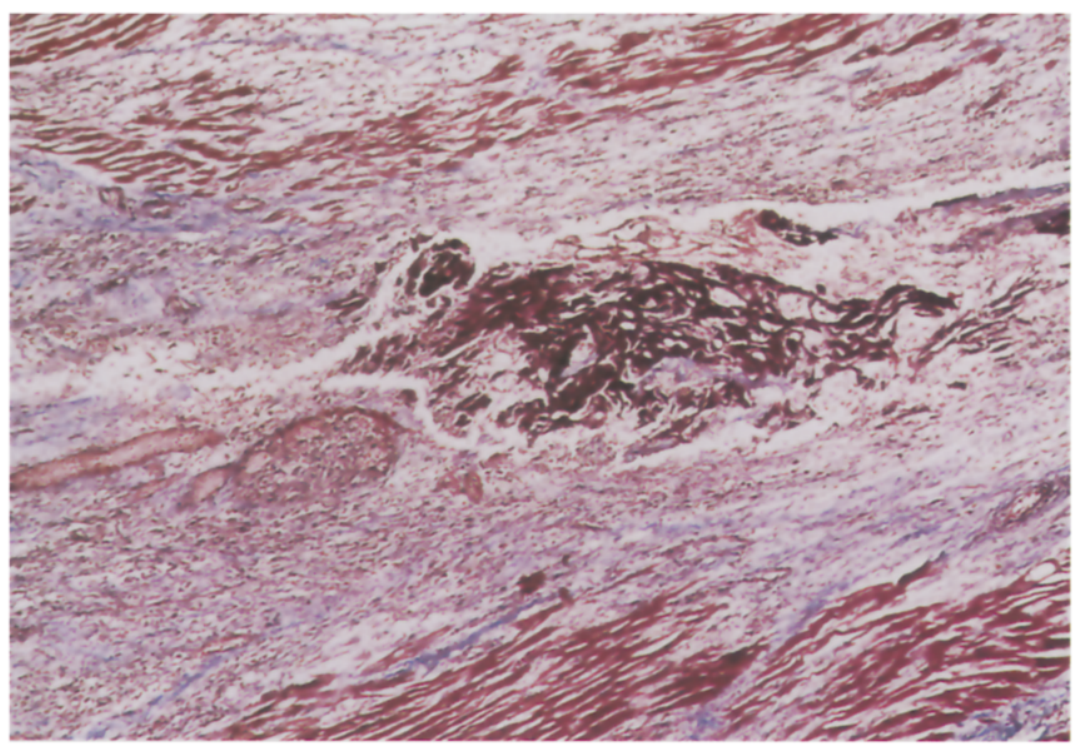

Fig. 1. Typical histologic appearance of a holmium:YAG TMLR channel 2 weeks after surgery. The original channel is infiltrated with granulation tissue with associated vascularity. The remaining central core is filled with organized thrombus indicating lack of active blood flow. Normal myocardium is seen staining deep red on the top and bottom edges of the figures. This appearance is similar to what has been observed in experimental animals at 2 weeks.

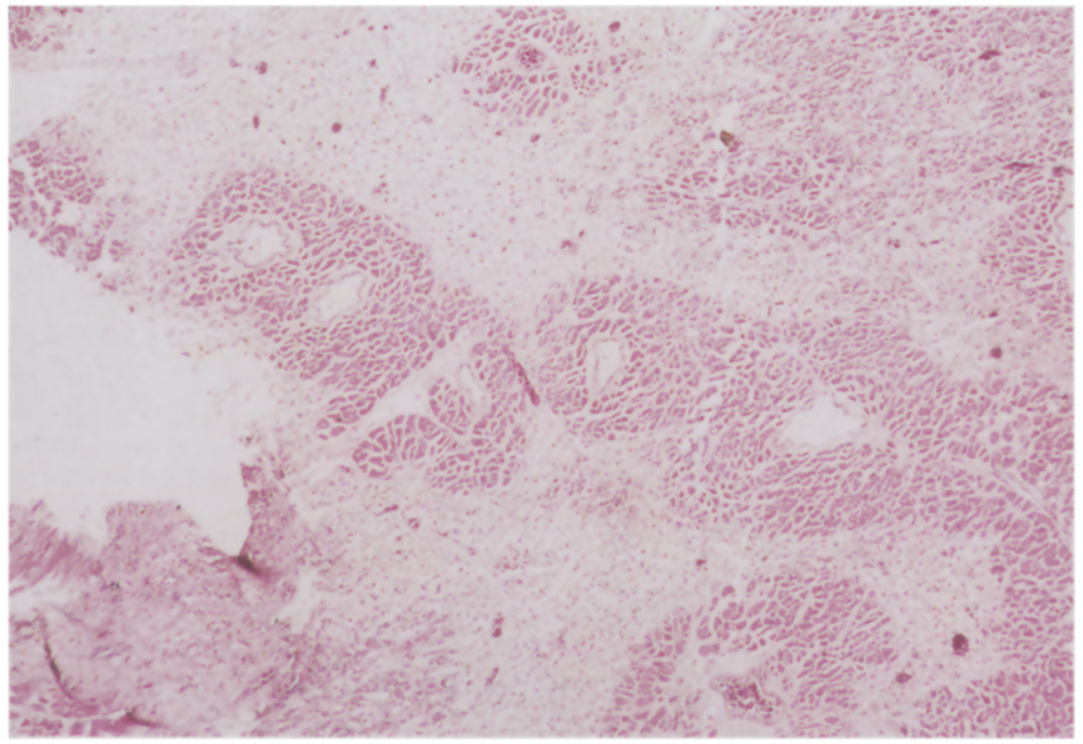

Fig. 2. Naturally occurring subendocardial, endothelium-lined sinusoids with surrounding zones of viable myocardium in the setting of surrounding myocardial infarction. Previous studies have documented ventricular chamber connections with these sinusoids, and the pattern of myocardial viability implies the extent to which direct perfusion from the ventricular chamber may contribute to myocardial perfusion. See text for further details.

sinusoids. Although these may represent dilated subendocardial veins that communicate with the epicardial circulation, direct connection to the ventricular chamber is strongly suggested by the fact that these patches exist in the setting of dense surrounding infarction. This setting indicates that despite severe impedance to epicardial blood flow to the region at some point, cells around these structures maintained viability, presumably owing to nu- 
tritive perfusion from an alternate source. These channels are not laser-made channels, because their density is much higher than the laser channel density and they lack the usual surrounding thermal damage.*

It has recently been shown that the endocardial region of alligator hearts has a dual blood supply consisting of an interconnecting epicardial circulation and direct connections to the ventricular chamber. The specific anatomic features of alligator hearts that permit a high degree of direct perfusion from the ventricular chamber appear to be their extensively branching network of large, densely packed channels, which creates thin sheets of myocardium ( $\leq 8$ cells thick) with relatively short myocardial distances from the channel edges and high surface area for nutrient and waste exchange. ${ }^{2}$ The density of subendocardial channels and the thickness of myocardium perfused via the naturally occurring subendocardial sinusoids observed in this patient are both reminiscent of this reptilian cardiac physiology. Accordingly, the present findings reveal the physiologic relevance (in terms of ability to nourish myocardium) of the previously recognized subendocardial vascular network known to communicate with both epicardial vessels and the ventricular chamber in human hearts. Specifically, it suggests that, as in alligator hearts, although myocardium can be perfused by this alternate mechanism, the amount of myocardium that can be perfused is limited.

As observed in this patient and in the experimental setting," the appearance of TMLR channels 2 weeks after surgery (i.e., channel remnants) is very different from that of both alligator heart channels and the naturally occurring sinusoids described herein. This strikingly different appearance of channel remnants suggests that mechanisms other than direct myocardial perfusion by blood flowing through truly patent permanent channels may underlie the clinical benefits of TMLR. Further evidence for lack of substantial direct myocardial perfusion in the

*Fisher PE, Kohmoto T, DeRosa CM, Spotnitz HM, Smith CR, Burkhoff D. Histologic analysis of transmyocardial laser channels: comparison of acute and chronic effects of different lasers. Unpublished data. subacute setting is provided by the fact that, as in this patient, TMLR channels fail to protect myocardium from postoperative myocardial infarction. Myocardial infarction is emerging as the major cause of postoperative morbidity and mortality after TMLR. On the other hand, results of two independent clinical studies with a carbon dioxide laser have documented an increase in regional blood flow by 3 months after the therapy. ${ }^{1,4}$ Consistent with these clinical studies, results of recent studies in animals suggest that one possible contributing mechanism may relate to stimulation of vascular growth after myocardial laser treatment. ${ }^{5}$ These findings, coupled with the observed average two-class reduction in angina at 3 months, which improves further over the course of the first postoperative year, suggests that this therapy provides benefits that cannot be explained by either placebo or thoracotomy effects. These types of encouraging results will continue to fuel a great deal of interest in a thorough evaluation of the clinical efficacy and search for the mechanisms underlying TMLR.

\section{REFERENCES}

1. Coley DA, Frazier OH, Kadipasaoglu KA, et al. Transmyocardial laser revascularization: clinical experience with twelve-month follow-up. J Thorac Cardiovasc Surg 1996; 111:791-9.

2. Kohmoto T, Argenziano M, Vliet KA, et al. Assessment of transmyocardial perfusion in alligator hearts [abstract]. Circulation 1996;94(Suppl):I295.

3. Burkhoff D, Fisher PE, Apfelbaum M, Kohmoto T, DeRosa CM, Smith CR. Histologic appearance of transmyocardial laser channels after 4-1/2 weeks. Ann Thorac Surg 1996;61: 1532-5.

4. Horvath KA, Mannting F, Cummings N, Shernan SK, Cohn LH. Transmyocardial laser revascularization: operative techniques and clinical results at two years. $\mathbf{J}$ Thorac Cardiovasc Surg 1996;111:1047-53.

5. Kohmoto T, Fisher PE, DeRosa C, Smith CR, Burkhoff D. Evidence of angiogenesis in regions treated with transmyocardial laser revascularization [abstract]. Circulation 1996; 94(Suppl):I294. 